\title{
Associations between Gilbert's syndrome and personality characteristics
}

\author{
Tolga Düzenli, ${ }^{1}$ (D) Özgür Maden, ${ }^{2}$ (D) Alpaslan Tanoğlu, ${ }^{3}$ (i) Mustafa Kaplan, ${ }^{4}$ (D) Yusuf Yazgan $^{3}$ (i)
}

\begin{abstract}
Objective: Gilbert's syndrome (GS) is a benign genetic disorder that is characterized by intermittent mild jaundice in which the liver doesn't process bilirubin properly. The aim of this study was to determine whether GS patients have a different personality structure and if there are associations between properties of temperament and character and total bilirubin levels.

Methods: A total of 1665 young male individuals aged from 19 to 30 who were admitted for occupational examinations were included in this study. Careful patient history was taken, a detailed physical examination was conducted, and hematologic and biochemical tests and abdominal ultrasonography were performed. The Turkish version of the Temperament and Character Inventory (TCI) was administered to all participants. 81 patients diagnosed with GS and 150 randomly chosen healthy individuals (control group) were investigated with comparison and correlation analyses.

Results: GS patients had higher scores than healthy controls for disorderliness (NS4) $(p=0.018)$, sentimentality (RD1) $(p=0.042)$, and fatigability (HA4) $(p=0.03)$. Moreover, Gilbert syndrome patients scored lower than controls for empathy $(\mathrm{C} 2)(\mathrm{p}=0.041)$ and transpersonal identification $(\mathrm{ST} 2)(\mathrm{p}=$ $0.044)$. Bilirubin levels were positively associated with disorderliness (NS4) $(r=0.141, p=0.032)$ and fatigability (HA4) $(r=0.14, p=0.033)$.

Conclusions: GS patients may have some different personality characteristics from healthy individuals. This study is an initial exploration of the personality structure of GS patients and the findings should be interpreted with caution. Further prospective studies are needed to identify the relationship between Gilbert disease and personality characteristics.
\end{abstract}

Keywords: Gilbert's syndrome, Temperament and Character İnventory, personality profile.

\section{Introduction}

Gilbert's syndrome (GS) is a benign genetic disorder that is characterized as intermittent mild jaundice, in which the liver doesn't process bilirubin properly. The mechanism of GS is linked to reduced uridine diphosphate-glucuronyl-transferase (UGT) $1 \mathrm{~A} 1$ activity, resulting in unconjugated hyperbilirubinemia. ${ }^{1}$
Unconjugated hyperbilirubinemia occurs intermittently when patients are subject to stress factors, such as physical stress, prolonged fasting, and/or poor diet. ${ }^{1}$ Emotional stimuli are associated with increases in the oxidative metabolites of bilirubin in human urine. ${ }^{2}$ On the other hand, GS is believed to reduce the risk of various diseases because of the antioxidant properties of bilirubin. ${ }^{3}$

\footnotetext{
1 Department of Gastroenterology, Hitit University Erol Olcok Training and Research Hospital, Corum, Turkey. 2 Department of Psychiatry, Sultan Abdulhamid Han Training and Research Hospital, Istanbul, Turkey. 3 Department of Gastroenterology, Sultan Abdulhamid Han Training and Research Hospital, Istanbul, Turkey. ${ }^{4}$ Department of Internal Medicine, Sultan Abdulhamid Han Training and Research Hospital, Istanbul, Turkey.

Submitted Feb 09 2020, accepted for publication Sep 212020.

Suggested citation: Düzenli T, Maden O, Tanoğlu A, Kaplan M, Yazgan Y. Associations between Gilbert's syndrome and personality characteristics. Trends Psychiatry Psychother. 2021;43(2):151-158. http://dx.doi.org/10.47626/2237-6089-2020-0003
} 
Although associations between emotional stimuli and increased oxidative metabolites of bilirubin in human urine have been demonstrated, it is not obvious whether GS patients have a different personality structure or whether there are associations between properties of temperament and character and total bilirubin levels.

The dimensional method has utility for personality assessment according to Cloninger's psychobiological model. Personality has been modeled as two discrete components: character and temperament. Temperament is defined as a constitutionally or biologically based segment of the personality, expresses the automatic sentimental reaction to events, and is influenced by emotional, motor, and attentional reactivity and self-regulation, underlying a variety of personal decisions. ${ }^{4,5}$

Character is defined as a self-concept, and is influenced by our interactions with people and our experiences and facility for learning. Therefore, these interactions and experiences enable greater flexibility and thus organization of personal differences in values and intentions.

Cloninger hypothesized that neurotransmitters were related to behavioral manifestations. ${ }^{4}$ In this context, as an inherited disorder, GS could be associated with altered glucuronidation rates of these metabolites and, consequently, with behavioral manifestations.

The aim of this study was to investigate associations with temperament and character properties in GS patients and healthy individuals.

\section{Methods}

\section{Study design and participants}

A total of 1665 young male individuals aged from 19 to 30 who were admitted to our hospital for occupational examinations were included in this retrospective, cross-sectional study. The study was approved by the institutional ethics committee and was therefore performed in accordance with the ethical standards laid down in the 1964 Helsinki Declaration and its later amendments. The patients included in the study provided written consent. Patient history was taken with care, a detailed physical examination was conducted, and hematologic and biochemical tests and abdominal ultrasonography were performed. All participants were examined by the departments of internal medicine, general surgery, cardiology, otorhinolaryngology, dermatology, ophthalmology, orthopedics, neurology, and psychiatry. After 12-hour overnight fasting, blood samples were obtained from patients' brachial veins in the biochemistry department and tested in approximately 1-2 hours.

In addition to total and direct/indirect bilirubin levels; hemogram, alanine aminotransferase (ALT), aspartate aminotransferase (AST), alkaline phosphatase (ALP), gamma glutamyl transferase (GGT), lactate dehydrogenase (LDH), glucose, urea, creatinine, sodium, potassium, lipid panel (HDL-cholesterol, LDL-cholesterol, total cholesterol, triglyceride), sedimentation rate, thyroid stimulating hormone (TSH), vitamin b12, folate, ferritin, albumin, and hepatitis markers (HbsAg, anti$\mathrm{HCV}$, anti-HIV) were measured.

Patients with alcohol/narcotics use, body mass index $>30 \mathrm{~kg} / \mathrm{m}^{2}$, abnormal test results (other than indirect/total bilirubin elevation), or any known history of disease or drug use were excluded from the study ( $n$ $=683$ ). A total of 982 people were eligible according to these criteria (Figure 1). Of these, 81 patients who had a total bilirubin value of $1.4 \mathrm{mg} / \mathrm{dL}$ or more, with no evidence of hemolysis or other pathological results (all of the tests mentioned above, including normal hemogram parameters, normal cholestatic enzymes, negative serological markers of viral hepatitis, and normal abdominal ultrasonography) were diagnosed as having GS. ${ }^{1,6}$ A control group was formed by randomly selecting 150 patients from the remaining 901 patients. These two groups were investigated by comparison and correlation analyses.

\section{Personality characteristics}

All study participants were instructed to fill in the Turkish version of the TCI for personality assessment. 4,5,7 Temperament is divided into four different independent parts in this representation, as follows: novelty seeking (NS), harm avoidance (HA), reward dependence (RD), and persistence (PS). In turn, NS consists of exploratory excitability (NS1), impulsiveness (NS2), extravagance (NS3), and disorderliness (NS4); HA contains the elements anticipatory worry (HA1), fear of uncertainty (HA2), shyness (HA3), and fatigability (HA4); RD comprises sentimentality (RD1), openness to friendly communication (RD2), attachment (RD3), and dependence (RD4); and PS is a fourth independent dimension.

Character is made up of three dimensions; cooperativeness (C), self-directedness (SD), and self-transcendence (ST). In turn, $C$ includes social acceptance (C1), empathy (C2), helpfulness (C3), compassion ( $\mathrm{C} 4)$, and pure-hearted conscience (C5); SD contains responsibility (SD1), purposefulness (SD2), resourcefulness (SD3), self-acceptance (SD4), and congruence (SD5); and ST is made up of self- 
forgetfulness (ST1), transpersonal identification (ST2), and spiritual acceptance (ST3).

\section{Statistical analyses}

The Statistical Package for the Social Sciences (SPSS) version 15.0 was used for statistical analysis. Results with $\mathrm{p}<0.05$ were considered statistically significant. Descriptive properties of the study groups were expressed as mean \pm standard deviation (SD) or percentage $(\%)$ and number $(n)$. The means of the measurements for each patient were calculated and analyzed. The Kolmogorov-Smirnov test was used to determine conformity to normal distribution. The Mann Whitney $U$ test was used to compare variables which did not fit a normal distribution. We used chi-square tests to compare qualitative data. Pearson and Spearman tests were used for correlation analyses.

\section{Results}

There were 81 patients in the GS group and 150 patients in the control group. All of the patients were male. The mean age was $22.7 \pm 1.6$ years in the GS group and $23.1 \pm 1.6$ years in the control groups ( $p$ $>0.05)$. There were no differences between the two groups in terms of the following demographic variables: marital status, number of siblings or their order, where or with whom they were living, previous job experience, economic and family status, mothers' and fathers' health status or educational levels, or academic and school performance (Table 1).

The GS patients had significantly higher scores than the controls for disorderliness (NS4) ( $p=0.018)$, sentimentality (RD1) ( $p=0.042)$, and fatigability $($ HA4) $(p=0.03)$ (Table 2$)$. GS patients scored lower

Table 1 - Sociodemographic characteristics of Gilbert's syndrome patients and control subjects

\begin{tabular}{|c|c|c|c|}
\hline & Gilbert $(n=81)$ & Control $(n=150)$ & $\mathbf{P}$ \\
\hline Age, mean & $22.7 \pm 1.6$ & $23.1 \pm 1.6$ & 0.055 \\
\hline \multicolumn{4}{|l|}{ Marital status } \\
\hline Married & 0 & 3.3 & 0.165 \\
\hline Single & 100 & 96.7 & \\
\hline \multicolumn{4}{|l|}{ Siblings } \\
\hline Number & $2.4 \pm 1.4$ & $2.3 \pm 1.3$ & 0.856 \\
\hline Order & $1.9 \pm 1.1$ & $2.0 \pm 1.0$ & 0.384 \\
\hline \multicolumn{4}{|l|}{ Residence } \\
\hline Village & 8.6 & 14.7 & 0.252 \\
\hline Town & 18.5 & 12 & \\
\hline City & 42 & 48 & \\
\hline Metropolis & 30.9 & 25.3 & \\
\hline \multicolumn{4}{|c|}{ Living arrangements } \\
\hline Family & 37.0 & 35.3 & 0.392 \\
\hline Friend & 27.2 & 36.0 & \\
\hline Alone & 13.6 & 8.0 & \\
\hline Youth hostel & 22.2 & 20.7 & \\
\hline \multicolumn{4}{|l|}{ Job experience } \\
\hline Yes & 44.4 & 40 & 0.513 \\
\hline No & 55.6 & 60 & \\
\hline \multicolumn{4}{|l|}{ Economic status } \\
\hline Low & 16.0 & 18.0 & 0.912 \\
\hline Medium & 81.5 & 80.0 & \\
\hline High & 2.5 & 2.0 & \\
\hline
\end{tabular}


than controls for the empathy (C2) $(p=0.041)$ and transpersonal identification (ST2) dimensions ( $p=$ 0.044 ). There were no statistically significant differences between the two groups for any of the other parameters. The mean scores of the TCI dimensions for GS patients and control subjects are presented in Table 2 .

We also applied an effect size (ES) measure to the significance of the statistical differences. ES indicates that if two groups' means do not differ by at least 0.2 standard deviations, the difference is trivial, even if it is statistically significant. Our study groups' results are presented in Table 3.

There were low-positive significant correlations between total bilirubin levels and disorderliness (NS4) $(r=0.141, p=0.032)$ and fatigability (HA4) $(r=$ $0.14, p=0.033$ ) (Table 4). There were no significant correlations between the other TCI dimensions and patients' total bilirubin levels.

Table 1 (cont.)

\begin{tabular}{|c|c|c|c|}
\hline & Gilbert $(n=81)$ & Control $(n=150)$ & $\mathbf{P}$ \\
\hline \multicolumn{4}{|l|}{ Family status } \\
\hline Nuclear & 79.0 & 78.7 & 0.994 \\
\hline Extended & 17.3 & 17.3 & \\
\hline Other* & 3.7 & 4.0 & \\
\hline \multicolumn{4}{|l|}{ Mother-ex/alive } \\
\hline Deceased & 1.2 & 2 & 0.562 \\
\hline Alive & 98.8 & 98 & \\
\hline \multicolumn{4}{|l|}{ Father-ex/alive } \\
\hline Deceased & 3.7 & 6.7 & 0.551 \\
\hline Alive & 96.3 & 93.3 & \\
\hline \multicolumn{4}{|c|}{ Education-mother } \\
\hline Uneducated & 12.3 & 5.3 & 0.302 \\
\hline Elementary & 58.0 & 64.7 & \\
\hline Middle school & 16.0 & 16.7 & \\
\hline High school & 12.3 & 10.0 & \\
\hline College & 1.2 & 3.3 & \\
\hline \multicolumn{4}{|l|}{ Education-father } \\
\hline Uneducated & 1.2 & 1.3 & 0.176 \\
\hline Elementary & 50.6 & 44.0 & \\
\hline Middle school & 25.9 & 18.7 & \\
\hline High school & 11.1 & 24.0 & \\
\hline College & 11.1 & 12.0 & \\
\hline \multicolumn{4}{|l|}{ Family income } \\
\hline Lower & 16.0 & 12.7 & 0.114 \\
\hline Middle & 81.5 & 87.3 & \\
\hline Upper & 2.5 & 0 & \\
\hline \multicolumn{4}{|c|}{ Academic/school performance } \\
\hline Very good & 13.6 & 8.0 & 0.546 \\
\hline Good & 54.3 & 61.3 & \\
\hline Not bad & 30.9 & 29.3 & \\
\hline Bad & 1.2 & 1.3 & \\
\hline
\end{tabular}

Data presented as percentages, unless otherwise specified. *Including stepfamilies; single-parent families, and other configurations not classified as nuclear or extended families.

154 - Trends Psychiatry Psychother. 2021;43(2) 
Table 2 - Mean TCI dimension scores for Gilbert's syndrome patients and control subjects

\begin{tabular}{|c|c|c|c|}
\hline & Gilbert $(n=81)$ & Control $(n=150)$ & $\mathbf{p}$ \\
\hline Total novelty seeking (NS) score & $15.6 \pm 3.0$ & $15.2 \pm 3.2$ & 0.204 \\
\hline Exploratory excitability (NS1) & $7.1 \pm 1.5$ & $7.1 \pm 1.5$ & 0.944 \\
\hline Impulsiveness (NS2) & $2.2 \pm 1.4$ & $2.3 \pm 1.5$ & 0.900 \\
\hline Extravagance (NS3) & $3.5 \pm 1.3$ & $3.4 \pm 1.4$ & 0.463 \\
\hline Disorderliness (NS4) & $2.8 \pm 1.4$ & $2.3 \pm 1.4$ & $0.018^{*}$ \\
\hline Total harm avoidance (HA) score & $7.7 \pm 4.3$ & $7.2 \pm 4.0$ & 0.409 \\
\hline Anticipatory worry (HA1) & $2.7 \pm 1.8$ & $2.6 \pm 1.8$ & 0.541 \\
\hline Fear of uncertainty (HA2) & $2.4 \pm 1.3$ & $2.3 \pm 1.4$ & 0.751 \\
\hline Shyness (HA3) & $1.0 \pm 1.3$ & $1.1 \pm 1.4$ & 0.466 \\
\hline Fatigability (HA4) & $1.6 \pm 1.3$ & $1.3 \pm 1.4$ & $0.030 *$ \\
\hline Total reward dependence (RD) score & $14.8 \pm 3.3$ & $14.7 \pm 2.7$ & 0.901 \\
\hline Sentimentality (RD1) & $6.4 \pm 1.6$ & $5.9 \pm 1.5$ & $0.042 *$ \\
\hline Attachment (RD3) & $5.3 \pm 1.7$ & $5.6 \pm 1.5$ & 0.141 \\
\hline Dependence (RD4) & $3.1 \pm 1.3$ & $3.2 \pm 1.4$ & 0.476 \\
\hline Persistence score & $6.7 \pm 1.2$ & $6.4 \pm 1.2$ & 0.551 \\
\hline Total self-directedness (SD) score & $34.0 \pm 5.0$ & $34.3 \pm 4.7$ & 0.610 \\
\hline Responsibility (SD1) & $6.3 \pm 1.5$ & $6.6 \pm 1.4$ & 0.267 \\
\hline Purposefulness (SD2) & $6.3 \pm 0.9$ & $6.4 \pm 1.0$ & 0.590 \\
\hline Resourcefulness (SD3) & $4.2 \pm 0.9$ & $4.2 \pm 1.0$ & 0.464 \\
\hline Self-acceptance (SD4) & $6.7 \pm 2.4$ & $6.9 \pm 2.2$ & 0.821 \\
\hline Congruence (SD5) & $10.4 \pm 1.4$ & $10.3 \pm 1.4$ & 0.511 \\
\hline Total cooperativeness (C) score & $35.0 \pm 3.9$ & $35.4 \pm 4.2$ & 0.313 \\
\hline Social acceptance (C1) & $7.2 \pm 1.0$ & $7.3 \pm 1.0$ & 0.416 \\
\hline Empathy (C2) & $5.4 \pm 1.1$ & $5.7 \pm 1.2$ & $0.041 *$ \\
\hline Helpfulness (C3) & $5.8 \pm 1.4$ & $5.8 \pm 1.3$ & 0.897 \\
\hline Compassion (C4) & $8.9 \pm 1.4$ & $9.0 \pm 1.3$ & 0.674 \\
\hline Pure-hearted conscience (C5) & $7.6 \pm 1.1$ & $7.5 \pm 1.1$ & 0.548 \\
\hline Total self-transcendence (ST) score & $17.5 \pm 4.8$ & $18.3 \pm 5.2$ & 0.385 \\
\hline Self-forgetfulness (ST1) & $4.7 \pm 1.7$ & $4.9 \pm 1.9$ & 0.550 \\
\hline Transpersonal identification (ST2) & $6.0 \pm 1.9$ & $6.5 \pm 1.9$ & $0.044 *$ \\
\hline Spiritual acceptance (ST3) & $6.7 \pm 2.8$ & $6.9 \pm 2.8$ & 0.800 \\
\hline
\end{tabular}

TCI = Temperament and Character Inventory.

$* p<0.05$ is considered statistically significant.

Table 3 - Associations between TCI and bilirubin levels

\begin{tabular}{lcccc}
\hline & Gilbert $(\mathbf{n}=\mathbf{8 1})$ & Control $(\mathbf{n}=\mathbf{1 5 0})$ & p & Effect size (Cohen's d) \\
\hline Disorderliness (NS4) & $2.8 \pm 1.4$ & $2.3 \pm 1.4$ & $0.018^{*}$ & 0.4 \\
Fatigability (HA4) & $1.6 \pm 1.3$ & $1.3 \pm 1.4$ & $0.030^{*}$ & 0.2 \\
Sentimentality (RD1) & $6.4 \pm 1.6$ & $5.9 \pm 1.5$ & $0.042^{*}$ & 0.4 \\
Empathy (C2) & $5.4 \pm 1.1$ & $5.7 \pm 1.2$ & $0.041^{*}$ & 0.3 \\
Transpersonal identification (ST2) & $6.0 \pm 1.9$ & $6.5 \pm 1.9$ & $0.044^{*}$ & 0.3 \\
\hline
\end{tabular}

TCI = Temperament and Character Inventory.

$\mathrm{d}=0.2$ is considered a 'small' effect size, 0.5 represents a 'medium' effect size, and 0.8 is a 'large' effect size. 
Table 4 - Associations between TCI and total bilirubin levels

\begin{tabular}{|c|c|c|c|c|c|c|c|c|}
\hline Variable & $\mathbf{r}$ & $\mathbf{P}$ & Variable & $\mathbf{r}$ & $\mathbf{p}$ & Variable & $\mathbf{r}$ & $\mathbf{p}$ \\
\hline NS total & 0.111 & 0.092 & RD total & -0.008 & 0.901 & C total & -0.058 & 0.383 \\
\hline NS1 & 0.028 & 0.669 & RD1 & 0.112 & 0.090 & $\mathrm{C} 1$ & -0.057 & 0.385 \\
\hline NS2 & 0.000 & 0.998 & RD3 & -0.074 & 0.264 & $\mathrm{C} 2$ & -0.116 & 0.077 \\
\hline NS3 & 0.072 & 0.273 & RD4 & 0.063 & 0.339 & $\mathrm{C} 3$ & -0.009 & 0.887 \\
\hline \multirow[t]{2}{*}{ NS4 } & 0.141 & $0.032 *$ & & & & $\mathrm{C} 4$ & 0.029 & 0.661 \\
\hline & & & & & & $\mathrm{C} 5$ & -0.001 & 0.985 \\
\hline HA total & 0.014 & 0.832 & SD total & -0.015 & 0.826 & ST total & -0.023 & 0.723 \\
\hline HA1 & 0.024 & 0.718 & SD1 & -0.087 & 0.186 & ST1 & 0.013 & 0.840 \\
\hline $\mathrm{HA} 2$ & -0.018 & 0.787 & SD2 & 0.016 & 0.811 & ST2 & -0.088 & 0.181 \\
\hline HA3 & -0.073 & 0.271 & SD3 & 0.008 & 0.901 & ST3 & -0.011 & 0.864 \\
\hline \multirow[t]{2}{*}{ HA4 } & 0.140 & $0.033^{*}$ & SD4 & -0.038 & 0.569 & & & \\
\hline & & & SD5 & 0.083 & 0.211 & & & \\
\hline $\mathrm{P}$ & 0.000 & 0.996 & & & & & & \\
\hline
\end{tabular}

$* \mathrm{p}<0.05$ is considered statistically significant.

NS = novelty seeking; NS1= exploratory excitability; NS2 = impulsiveness; NS3 = extravagance; NS4 = disorderliness; HA = harm avoidance; HA1 = anticipatory worry; HA2 = fear of uncertainty; HA3 = shyness; HA4 = fatigability; RD = reward dependence; RD1 = sentimentality; RD3 = attachment; RD4 = dependence; $\mathrm{P}=$ persistence; $\mathrm{SD}=$ self-directedness; SD1 = responsibility; SD2 = purposefulness; SD3 = resourcefulness; SD4 = self-acceptance; SD5 = congruence; $\mathrm{C}=$ cooperativeness; $\mathrm{C} 1=$ social acceptance; $\mathrm{C} 2=$ empathy; $\mathrm{C} 3=$ helpfulness; $\mathrm{C} 4=$ compassion; $\mathrm{C} 5=$ pure-hearted conscience; $\mathrm{ST}=$ selftranscendence; ST1 = self-forgetfulness; ST2 = transpersonal identification; ST3 = spiritual acceptance.

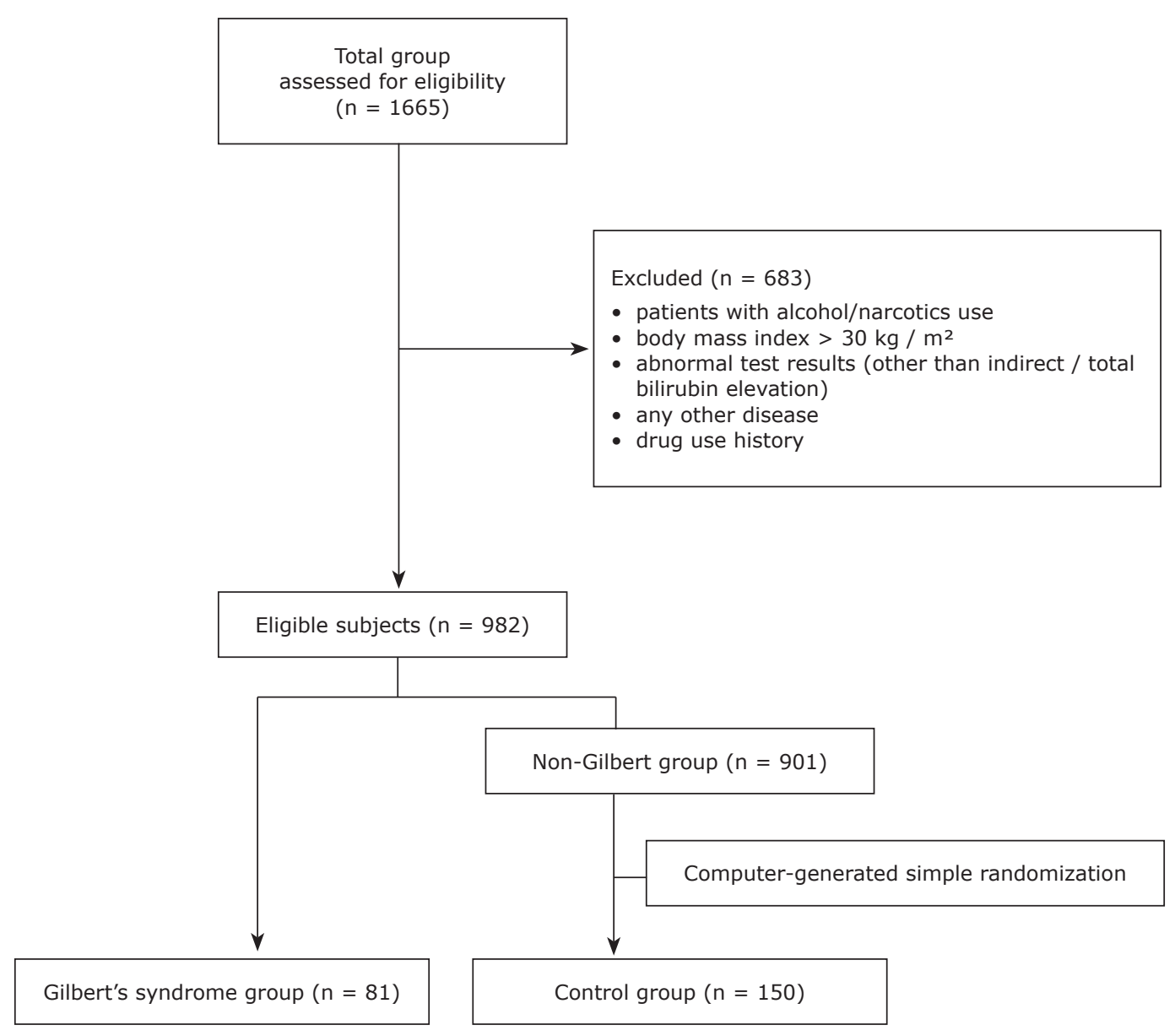

Figure 1 - Patient enrollment and classification. 


\section{Discussion}

The Temperament and Character Inventory-240 (TCI-240) has not been used with GS patients up to the present. Our current study showed that GS patients have a characteristic personality profile with higher scores for disorderliness, sentimentality, and fatigability and lower scores for empathy and transpersonal identification than healthy individuals.

Stress factors are associated with increased bilirubin and its metabolites in urine. Urinary excretion of bilirubin oxidative metabolites increases in septic patients. ${ }^{8}$ Novío et al. ${ }^{9}$ reported that stressed mice had higher oxidative metabolites of bilirubin than non-stressed mice and observed that levels declined after alprazolam doses were given to the stressed animals.

On the other hand, bilirubin has important antioxidant properties, which has been confirmed over the last 2 decades, contributing to defense against increased oxidative stress. Experimental and also clinical studies have indicated associations between low bilirubin concentrations and cardiovascular diseases, diabetes mellitus, certain cancers, autoimmune diseases such as lupus erythematosus or rheumatoid arthritis, and psychiatric disorders such as schizofrenia. ${ }^{7}$ Subjects with mildly elevated blood bilirubin levels, typical of GS, have decreased risk of these diseases. ${ }^{10}$ Maruhashi et al. ${ }^{11}$ evaluated the role of oxidative stress in endothelial function in patients with GS under normal conditions without cardiovascular risk factors. Patients with GS had low levels of oxidative stress associated with hyperbilirubinemia and enhanced endotheliumdependent vasodilation. ${ }^{11}$

The relationship between bilirubin levels and psychopathology has been investigated in a limited number of studies. Yamaguchi et al. ${ }^{2}$ indicated that emotional stimuli are associated with an increase in oxidative metabolites of bilirubin in human urine. Miyaoka et al. ${ }^{12}$ reported that bilirubin oxidative metabolites (biopyrrins) are increased in urine from patients with psychiatric disorders (schizophrenia and depression). Bilirubin concentration also exhibited correlation with positive symptoms in patients with schizophrenia. ${ }^{13}$ Tang et al. ${ }^{14}$ demonstrated that high bilirubin level is associated with post-stroke depression. These findings suggest that behavioral states are associated with increases in the oxidative metabolites of bilirubin in human urine. Less clear and still under study is the possible relation between serum bilirubin levels and neurological diseases. ${ }^{15}$ Kao et al. ${ }^{16}$ investigated the relationship between serum total bilirubin levels and functional dependence in older adults, revealing that higher serum total bilirubin levels were associated with lower likelihood of functional dependence. Oren et al. ${ }^{17}$ reported that nocturnal bilirubin levels were lower in seasonal depression patients than in controls. However, notwithstanding these neurochemical mechanisms, the social repercussions of a possible clinical manifestation with GS should not be overlooked. For example, repercussions of the stigma caused by chronic/ intermittent jaundice of individuals with GS may also have an effect on personality characteristics.

With regard to temperament, individuals who score low on the disorderliness subscale have a tendency to be disorganized, disordered, chaotic, and unsystematic. They do not like activities with strict rules and regulations. ${ }^{4,5}$

High harm avoidance scores are related to depression and anxiety symptoms, but there was no difference in harm avoidance parameters except fatigability. Although there is no consensus on fatigue as a symptom of GS, higher fatigability may be an expected outcome for GS patients. ${ }^{18,19}$ Moreover, with a significant low correlation between serum bilirubin levels and fatigability, our study may have revealed new evidence on the subject of fatigability for GS patients.

Reward dependence reflects behavior response, which stimulates a social reward and reflects a genetic tendency that stimulates continuation of this behaviour. ${ }^{4,5}$ In the present study, scores for the reward dependence subscale sentimentality were higher among patients with GS than in the control group. Significantly higher sentimentality scores in patients with GS may be due to placing more importance on social consent and to being susceptible or easily impressionable by others.

Character consists of three parts; cooperativeness (C), self-directedness (SD), and self-transcendence (ST). Within these, our study demonstrated lower scores for empathy and transpersonal identification in GS patients. Empathy is described as a sensation of unity or identification with other individuals and is said to enable improved communication and compassion for others. ${ }^{4,5}$ Transpersonal identification is related to spiritual acceptance or to apprehension of relationships that cannot be explained by analytical reasoning or demonstrated to others by objective observations. ${ }^{4,5}$

Cloninger hypothesized that neurotransmitters were related with behavioral manifestations, such as, for example, serotonin with harm avoidance (behavioral inhibition); norepinephrine with reward dependence (behavioral maintenance); dopamine with novelty seeking (behavioral activation), and glutamine with persistence (behavioral perseverance). ${ }^{4,5}$ In this context, as an inherited disorder, GS may be associated with altered glucuronidation rates of these metabolites. Lee et al. ${ }^{20}$ proposed that in a subgroup of Gilbert's 
syndrome patients, homozygosity for dual UGT $1 \mathrm{~A} 1$ and UGT 1 A6 polymorphisms may lead to altered metabolism and elimination of serotonin. Peters et al. ${ }^{21}$ mentioned that UGTs play important roles in mediating biological activity of endogenous substrates in addition to their roles in drug distribution, metabolism, and excretion processes.

This study can be considered an initial exploration of the personality structure of GS patients. However, we believe that clinicians should be cautious about interpreting the findings, in view of the study's confounding factors and limitations. Firstly, the size of the sample was relatively small for extrapolation of results. More studies with different psychological testing methods could be used to generalize our findings. Secondly, our study was a retrospective cross-sectional study. Further prospective studies are required to identify the causality of the links between Gilbert's disease and personality characteristics. Thirdly, the TCI is a self-report questionnaire and may be influenced by environmental factors. Fourthly, all the subjects were male and young, but this may also have ensured greater homogeneity of the groups and exclusion of age and gender effects on TCI results. Additionally, GS was diagnosed from clinical and laboratory parameters in this study, whereas definitive diagnosis can be made by genetic mutation analysis. Finally, the detailed results of medical departments' clinical and psychiatric evaluations were not available, only their final assessments and decisions, which could constitute a potential confounding factor between groups. Nevertheless, the study also included subjects who were assessed as 'healthy' by each of the departments. Subjects with any pathological conditions were excluded from the study.

In conclusion, our findings demonstrate significant differences in personality features (TCI 240) between GS patients and healthy individuals. There might be a relationship between GS and personality characteristics and therefore patients' personality features might merit attention when evaluating patients with GS. On the other hand, our findings were statistically significant, but the effect size was small and there are some confounding factors and limitations. Further prospective studies are needed to identify the relationship between Gilbert's disease and personality characteristics.

\section{Disclosure}

No conflicts of interest declared concerning the publication of this article.

\section{References}

1. Fretzayas A, Moustaki M, Liapi O, Karpathios T. Gilbert syndrome. Eur J Pediatr. 2012;171:11-5.

2. Yamaguchi T, Shioji I, Sugimoto A, Yamaoka M. Psychological stress increases bilirubin metabolites in human urine. Biochem Biophys Res Commun. 2002;293:517-20.

3. Horsfall LJ, Nazareth I, Pereira SP, Petersen I. Gilbert's syndrome and the risk of death: a population-based cohort study. J Gastroenterol Hepatol. 2013;28:1643-7.

4. Cloninger CR, Przybeck TR, Svrakic DM, Wetzel RD. The Temperament and Character Inventory (TCI): a guide to its development and use. St. Louis: Center for Psychobiology of Personality, Washington University; 1994.

5. Kose S, Sayar K, Kalelioglu U, Aydin N, Celikel FC, Gulec H, et al. Normative data and factorial structure of the Turkish version of the Temperament and Character Inventory. Compr Psychiatry. 2009;50:361-8.

6. Hirschfield GM, Alexander GJ. Gilbert's syndrome: an overview for clinical biochemists. Ann Clin Biochem. 2006;43:340-3.

7. Cloninger CR, Svrakic DM, Przybeck TR. A psychobiological model of temperament and character. Arch Gen Psychiatry. 1993; 50:975-90.

8. Otani K, Shimizu S, Chijiiwa K, Yamaguchi K, Kuroki S, Tanaka M. Increased urinary excretion of bilirubin oxidative metabolites in septic patients: a new marker for oxidative stress in vivo. J Surg Res. 2001;96:44-9.

9. Novío S, Núñez MJ, Ponte CM, Freire-Garabal M. Urinary biopyrrins: potential biomarker for monitoring of the response to treatment with anxiolytics. Basic Clin Pharmacol Toxicol. 2012;111:206-10.

10. Vítek L. Relationship of bilirubin to diseases caused by increased oxidative stres. Vnitr Lek. 2013;59:618-21.

11. Maruhashi T, Soga J, Fujimura N, Idei N, Mikami S, Iwamoto $Y$, et al. Hyperbilirubinemia, augmentation of endothelial function, and decrease in oxidative stress in Gilbert syndrome. Circulation. 2012;126:598-603.

12. Miyaoka T, Yasukawa R, Yasuda H, Shimizu M, Mizuno S, Sukegawa $\mathrm{T}$, et al. Urinary excretion of biopyrrins, oxidative metabolites of bilirubin, increases in patients with psychiatric disorders. Eur Neuropsychopharmacol. 2005;15:249-52.

13. Widschwendter CG, Rettenbacher MA, Kemmler G, et al. Bilirubin concentration correlates with positive symptoms in patients with schizophrenia. J Clin Psychiatry. 2016;77:512-6.

14. Tang WK, Liang $\mathrm{H}$, Chu WC, Mok V, Ungvari GS, Wong KS. Association between high serum total bilirubin and post-stroke depression. Psychiatry Clin Neurosci. 2013;67:259-64.

15. Gazzin S, Vitek L, Watchko J, Shapiro SM, Tiribelli C. A Novel Perspective on the Biology of Bilirubin in Health and Disease. Trends Mol Med. 2016;22:758-68.

16. Kao TW, Chou CH, Wang CC, Chou CC, Hu J, Chen WL. Associations between serum total bilirubin levels and functional dependence in the elderly. Intern Med J. 2012;42:1199-207.

17. Oren DA, Desan PH, Boutros N, Anand A, Charney DS. Effects of light on low nocturnal bilirubin in winter depression: a preliminary report. Biol Psychiatry. 2002;51:422-5.

18. Cleary KJ, White PD. Gilbert's and chronic fatigue syndromes in men. Lancet. 1993;341:842.

19. Valesini G, Conti F, Priori R, Balsano F. Gilbert's syndrome and chronic fatigue syndrome. Lancet. 1993;341:1162-3.

20. Lee $P$, Jones $G$, Seibel MJ. Dual polymorphisms in UDPglucuronosyltransferases $1 \mathrm{~A} 1$ and 1A6: a novel mechanism for hyperserotoninaemia in Gilbert's syndrome mimicking carcinoid syndrome? Eur J Gastroenterol Hepatol. 2007;19:337-40.

21. Peters WH, te Morsche RH, Roelofs HM. Combined polymorphisms in UDP-glucuronosyltransferases $1 \mathrm{~A} 1$ and 1A6: implications for patients with Gilbert's syndrome. J Hepatol. 2003;38:3-8.

\section{Correspondence:}

Tolga Düzenli

Hitit University Erol Olcok Training and Research Hospital, Department of Gastroenterology

Çepni, İnönü Cd. No:176

19040 - Çorum - Turkey

Tel.: +903642193000

Fax.: +903642193030

E-mail: tolgaduzenli@yahoo.com 